\title{
Synchronization transitions in ensembles of noisy oscillators with bi-harmonic coupling
}

\author{
Vladimir Vlasov $^{1}$, Maxim Komarov ${ }^{1,2}$ and Arkady Pikovsky ${ }^{1,2}$ \\ ${ }^{1}$ Institute for Physics and Astronomy, University of Potsdam, D-14476 Potsdam, \\ Germany \\ ${ }^{2}$ Department of Control Theory, Nizhni Novgorod State University, Gagarin Av. 23, \\ 606950 Nizhni Novgorod, Russia
}

E-mail: mrvoov@gmail.com (V Vlasov)

Received 14 November 2014, revised 13 January 2015

Accepted for publication 16 January 2015

Published 12 February 2015

\begin{abstract}
We describe synchronization transitions in an ensemble of globally coupled phase oscillators with a bi-harmonic coupling function, and two sources of disorder-diversity of the intrinsic oscillators' frequencies, and external independent noise forces. Based on the self-consistent formulation, we derive analytic solutions for different synchronous states. We report on various nontrivial transitions from incoherence to synchrony, with the following possible scenarios: simple supercritical transition (similar to classical Kuramoto model); subcritical transition with large area of bistability of incoherent and synchronous solutions; appearance of a symmetric two-cluster solution which can coexist with the regular synchronous state. We show that the interplay between relatively small white noise and finite-size fluctuations can lead to metastability of the asynchronous solution.
\end{abstract}

Keywords: synchronization, bi-harmonic coupling, noise

(Some figures may appear in colour only in the online journal)

\section{Introduction}

In the theory of synchronization, the Kuramoto model of globally coupled phase oscillators $[1-3]$ is one of the most popular setups for describing synchronization phenomena. The case of a harmonic sin-coupling function is well studied in literature $[2,4,5]$. However, when reducing complex nonlinear oscillatory dynamics to relatively simple phase models, one often has to deal with multi-harmonic coupling functions [2]. In this paper we concentrate on the particular case of the Kuramoto model of globally coupled phase oscillators with a biharmonic interaction function. Such a type of interaction between oscillators arises in several 
realistic physical setups, including: (i) classical Huygens' setup with pendulum clocks suspended on a common beam with both vertical and horizontal displacements [6, 7]; (ii) recently experimentally observed $\varphi$-Josephson junctions, where the dynamics of a single junction in the array is governed by a double-well energy potential [8]; (iii) globally coupled electrochemical oscillators $[9,10]$, where the second harmonics has been identified from the experimental data.

In our work we take into account two main sources of disorder that hinder synchronization, namely diversity of oscillators' frequencies, and white additive noise acting on the phases. Recent theoretical studies indicate that in the noise-free case the Kuramoto model with bi-harmonic coupling function is characterized by a variety of multi-branch entrainment modes [11-13]. This variety manifests itself as a multiplicity (multistability) of different synchronous states, with distinct redistributions of oscillators between two stable branches of microscopic dynamics [7, 14]. In this work we show that the action of white noise removes the multiplicity; however, the overall picture of transitions from incoherence to synchrony is non-trivial and can be quite complex in comparison to the standard Kuramoto model.

The paper is organized as follows. First, we formulate the problem and perform a special, suitable for the analysis, parametrization of the system. Then, we present a self-consistent approach allowing us to find order parameters as a function of the coupling constants and the disorder in an analytic form. Wherever possible, we perform the stability analysis. And at last, we discuss the limiting case of small noise intensity and its relation to the noiseless situation. In conclusion, we summarize and collect all the findings together.

\section{Basic model}

In this paper we study an ensemble of phase oscillators (phase variables $\phi_{k}$ ), subject to a mean-field bi-harmonic coupling and noise (in this formulation we use 'primed' variables, which will be in short transformed to dimensionless ones):

$$
\frac{\mathrm{d} \phi_{k}}{\mathrm{~d} t^{\prime}}=\omega_{k} \Delta^{\prime}+\frac{\varepsilon}{N} \sum_{j=1}^{N} \sin \left(\phi_{j}-\phi_{k}\right)+\frac{\gamma}{N} \sum_{j=1}^{N} \sin \left(2 \phi_{j}-2 \phi_{k}\right)+\sqrt{D^{\prime}} \xi_{k}\left(t^{\prime}\right) .
$$

Here $\omega_{k}$ are normalized natural frequencies of oscillators that are assumed to have a symmetrical unimodal distribution $g(\omega)$ with unit width and zero mean value (the latter condition is not a restriction, as it can be always achieved by transforming to a properly rotating reference frame). Parameter $\Delta^{\prime}$ measures the spread of the distribution of natural frequencies. Gaussian white noise is defined according to $\left\langle\xi_{k}\left(t_{1}^{\prime}\right) \xi_{j}\left(t_{2}^{\prime}\right)\right\rangle=2 \delta\left(t_{1}^{\prime}-t_{2}^{\prime}\right) \delta_{k j}$. Parameters $\varepsilon$ and $\gamma$ define the strength of the coupling on the first and the second harmonics, respectively.

Equation (1) can be rewritten as

$$
\frac{\mathrm{d} \phi_{k}}{\mathrm{~d} t^{\prime}}=\omega_{k} \Delta^{\prime}+\varepsilon R_{1} \sin \left(\Theta_{1}-\phi_{k}\right)+\gamma R_{2} \sin \left(\Theta_{2}-2 \phi_{k}\right)+\sqrt{D^{\prime}} \xi_{k}\left(t^{\prime}\right)
$$

where $R_{m} \mathrm{e}^{\mathrm{i} \Theta_{m}}=N^{-1} \sum_{j} \mathrm{e}^{\mathrm{i} m \phi_{j}}, m=1,2$, are the two relevant order parameters [15].

Equation (2) has four parameters, all of them of dimension $1 / t^{\prime}: \Delta^{\prime}, \varepsilon, \gamma, D^{\prime}$. By rescaling time, one parameter can be set to one. We choose to set the overall coupling $\varepsilon+\gamma$ to unity. Thus, we introduce $t=(\varepsilon+\gamma) t^{\prime}$ and get

$$
\dot{\phi}_{k}=\omega_{k} \Delta+q R_{1} \sin \left(\Theta_{1}-\phi_{k}\right)+(1-q) R_{2} \sin \left(\Theta_{2}-2 \phi_{k}\right)+\sqrt{D} \xi_{k}(t),
$$


where $\Delta=\Delta^{\prime} /(\varepsilon+\gamma), q=\varepsilon /(\varepsilon+\gamma)$ and $D=D^{\prime} /(\varepsilon+\gamma)$. Parameter $q$ describes the relation between coupling coefficients $\varepsilon$ and $\gamma$, such that the case $q=0$ corresponds to a pure second harmonic coupling with $\varepsilon=0$, while $q=1$ corresponds to a pure Kuramoto-type first harmonic coupling with $\gamma=0$. In this new normalization, increasing or decreasing of coupling strength is equivalent to decreasing or increasing of the disorder parameters $\Delta$ (spread of frequencies) and $D$ (noise), while keeping a constant relation $\Delta / D$ between them. This suggests to introduce new parameters $T, s$ in such a way that $\Delta=(1-s) T, D=s T$. Therefore, parameter $T$ measures the overall disorder (noise plus spread of frequencies), normalized by the overall coupling strength $\varepsilon+\gamma$. Parameter $s$ measures the relation between parameters $\Delta$ (width of frequency distribution) and $D$ (noise): for $s=0$ the system is purely deterministic, while for $s=1$ it describes an ensemble of identical noisy oscillators. Summarizing, equation (3) with new parameters $q, T, s$ becomes

$\dot{\phi}_{k}=\omega_{k}(1-s) T+q R_{1} \sin \left(\Theta_{1}-\phi_{k}\right)+(1-q) R_{2} \sin \left(\Theta_{2}-2 \phi_{k}\right)+\sqrt{s T} \xi_{k}(t)$.

We consider the thermodynamic limit $N \rightarrow \infty$, then the order parameters are just ensemble averages $R_{m} \mathrm{e}^{\mathrm{i} \Theta_{m}}=\left\langle\mathrm{e}^{\mathrm{i} m \phi}\right\rangle$ and can be represented through the conditional probability density function of the phases $\rho(\varphi, t \mid \omega)$, as

$$
R_{m} \mathrm{e}^{\mathrm{i} \Theta_{m}}=\left\langle\mathrm{e}^{\mathrm{i} m \varphi}\right\rangle=\iint g(\omega) \rho(\varphi, t \mid \omega) \mathrm{e}^{\mathrm{i} m \varphi} \mathrm{d} \varphi \mathrm{d} \omega .
$$

In the thermodynamic-limit formulation we use the variable $\varphi$ to describe the phase and skip all indices; therefore, according to (4) the equation for the phase variable $\varphi$ at given $\omega$ reads

$$
\frac{\mathrm{d} \varphi}{\mathrm{d} t}=\omega(1-s) T+q R_{1} \sin \left(\Theta_{1}-\varphi\right)+(1-q) R_{2} \sin \left(\Theta_{2}-2 \varphi\right)+\sqrt{s T} \xi(t) .
$$

The Fokker-Planck equation for $\rho(\varphi, t \mid \omega)$ follows from equation (6):

$$
\frac{\partial \rho}{\partial t}+\frac{\partial}{\partial \varphi}\left[\left(\omega(1-s) T+q R_{1} \sin \left(\Theta_{1}-\varphi\right)+(1-q) R_{2} \sin \left(\Theta_{2}-2 \varphi\right)\right) \rho\right]=s T \frac{\partial^{2} \rho}{\partial \varphi^{2}}
$$

The limiting noise-free case when $s=0$ has been described in detail in [7, 14]. In this paper we will present a general analysis for systems with noise and a finite distribution of frequencies. We will treat the limit $s \ll 1$, which appears to be singular, separately. The other limiting case $s=1$ is the case of the identical natural frequencies, in terms of the analysis presented below it is not special. However, for $s=1$ an additional stability analysis can be performed, thus this case will be also considered in detail separately.

\section{Stationary solutions: self-consistent approach}

A disordered state with a uniform distribution of phases $\rho=(2 \pi)^{-1}$ and vanishing order parameters $R_{1}=R_{2}=0$ is always a solution of the system (5), (7). Additionally, we expect nontrivial synchronized states of two types: (i) all order parameters are non-zero, and (ii) a symmetric two-cluster distribution where all odd order parameters vanish $R_{2 m+1}=0$ and $R_{2 m} \neq 0, m \in \mathbb{N}_{0}$.

\subsection{Solution in a parametric form}

Due to the symmetry of the coupling function and of the distribution of natural frequencies $g(\omega)$, the nontrivial solutions are stationary states (which means that the frequency of the 
mean fields is exactly the average oscillator frequency), which can be obtained by setting $\Theta_{1}=\Theta_{2}=0$ (in fact, one of the phases of the order parameters is arbitrary, the condition above means that the phase shift between the two order parameters is zero). We discuss this setting in full detail in appendix A, where we also show how to describe asymmetric regimes with non-trivial frequency of the mean field and asymmetric coupling functions and frequency distribution. As in this paper we focus on the analysis of the symmetric setup, below we follow the derivation for this case.

In the symmetric setting, the stationary conditional probability density function $\rho(\varphi \mid \omega)$ satisfies the stationary Fokker-Planck equation

$$
\frac{\partial}{\partial \varphi}\left[\left(\omega(1-s)-\frac{q R_{1}}{T} \sin (\varphi)-\frac{(1-q) R_{2}}{T} \sin (2 \varphi)\right) \rho\right]=s \frac{\partial^{2} \rho}{\partial \varphi^{2}},
$$

where because of symmetry

$$
R_{m}=\iint g(\omega) \rho(\varphi \mid \omega) \cos (m \varphi) \mathrm{d} \varphi \mathrm{d} \omega
$$

To find the solutions of this self-consistent system explicitly, it is convenient to introduce two new auxiliary variables $R$ and $\alpha$ (together with definitions $u, v$, and $x$ ) according to

$$
\begin{aligned}
& R=\sqrt{\left(q R_{1} / T\right)^{2}+\left((1-q) R_{2} / T\right)^{2}}, \\
& u=\cos \alpha=\frac{q R_{1}}{T R}, \\
& v=\sin \alpha=\frac{(1-q) R_{2}}{T R}, \\
& x=\frac{\omega}{R} .
\end{aligned}
$$

Then the stationary Fokker-Planck equation (8) for the stationary distribution density $\rho(\varphi \mid x)$ (which depends on $R, \alpha, s$ as parameters) reads

$$
\frac{\partial}{\partial \varphi}[R(x(1-s)-u \sin (\varphi)-v \sin (2 \varphi)) \rho]=s \frac{\partial^{2} \rho}{\partial \varphi^{2}} .
$$

An explicit solution of (11) can be written as double integrals, but practically it is more convenient to solve it in the Fourier modes representation

$$
\rho(\varphi \mid x)=\frac{1}{2 \pi} \sum_{n} C_{n}(\alpha, R, s, x) \mathrm{e}^{\mathrm{i} n \varphi}, \quad C_{n}(\alpha, R, s, x)=\int_{0}^{2 \pi} \rho \mathrm{e}^{-\mathrm{i} n \varphi} \mathrm{d} \varphi, \quad C_{0}=1 .
$$

Substituting (12) in equation (11) we obtain

$$
\begin{aligned}
0 & =\int_{0}^{2 \pi}\left[-\frac{\partial}{\partial \varphi}[R(x(1-s)-u \sin (\varphi)-v \sin (2 \varphi)) \rho]+s \frac{\partial^{2} \rho}{\partial \varphi^{2}}\right] \mathrm{e}^{-\mathrm{i} k \varphi} \mathrm{d} \varphi \\
& =R\left[\left(-\mathrm{i} x(1-s) k-k^{2} s / R\right) C_{k}+\mathrm{i} k u \frac{C_{k-1}-C_{k+1}}{2 \mathrm{i}}+\mathrm{i} k v \frac{C_{k-2}-C_{k+2}}{2 \mathrm{i}}\right] .
\end{aligned}
$$

Thus, from (13) we obtain a system of linear algebraic equations for the mode amplitudes:

$$
2(\mathrm{i} x(1-s)+k s / R) C_{k}+u\left(C_{k+1}-C_{k-1}\right)+v\left(C_{k+2}-C_{k-2}\right)=0,
$$

which can be solved by standard methods after a proper truncation to a finite number of Fourier modes (which controls accuracy of the solution) is performed. After finding 
$C_{1,2}(\alpha, R, s, x)$, we have to calculate integrals

$$
F_{1,2}(\alpha, R, s)=\int g(R x) \operatorname{Re}\left[C_{1,2}(\alpha, R, s, x)\right] \mathrm{d} x .
$$

This allows us to represent the order parameters as

$$
R_{1,2}(\alpha, R, s)=R \int g(R x) \operatorname{Re}\left[C_{1,2}(\alpha, R, s, x)\right] \mathrm{d} x=R F_{1,2}(\alpha, R, s) .
$$

Substituting this in equation (10), we obtain our parameters $T, q$ as functions of the auxiliary variables

$$
\begin{aligned}
T & =\frac{1}{\frac{\cos \alpha}{F_{1}}+\frac{\sin \alpha}{F_{2}}}, \\
q & =\frac{\frac{\cos \alpha}{F_{1}}}{\frac{\cos \alpha}{F_{1}}+\frac{\sin \alpha}{F_{2}}}=\frac{1}{1+\frac{F_{1}}{F_{2}} \tan \alpha} .
\end{aligned}
$$

Summarizing, we have obtained the explicit solution of the self-consistent problem: for each fixed $s$, by varying $\alpha \in[0, \pi / 2]$ and $R \in[0, \infty)$, we obtain the solution in a parametric form: $R_{1,2}=R_{1,2}(\alpha, R)$ according to (16), $T=T(\alpha, R)$ and $q=q(\alpha, R)$ according to (17).

The case of purely two-cluster state with $R_{1}=0$ corresponds to $\alpha=\pi / 2$, it is singular in (16), (17). Here the solution is represented as

$$
R_{2}=R F_{2}, \quad T=(1-q) F_{2} .
$$

Thus, by the method presented above, it is possible to find stationary solutions of equation (7) for any given $q, T$ and $s$. In the general case of $s<1$, equation (7) is integrodifferential equation and the analysis of the stability of all solutions is quite difficult, except for the simplest incoherent solution $\rho=(2 \pi)^{-1}$. However, in the limiting case of identical natural frequencies $s=1$, density $\rho$ is $\omega$-independent, and integration in (9) over the frequencies gives always 1 . This means that Fourier modes (12) are in fact the order parameters: $R_{m}(q, T, 1)=\operatorname{Re}\left[C_{m}(q, T, 1,0)\right]$. The same is valid also for the full time-dependent problem: it can be written as a system of nonlinear ordinary differential equations for timedependent Fourier modes of the density, which can be analyzed for stability after a proper truncation. Thus, we start with this particular case.

\subsection{Limiting case of identical oscillators}

Here we describe the case of identical natural frequencies, which means that $\Delta=(1-s) T=0$ or $s=1$. As discussed above, in this case also a stability analysis is possible, which we outline below. First, let us represent equation (7) in terms of Fourier modes $C_{m}$ which are related to the complex order parameters $C_{m}^{*}=R_{m} \mathrm{e}^{\mathrm{i} \Theta_{m}}$ (the procedure is the same as in obtaining (13)):

$\frac{\mathrm{d} C_{k}}{\mathrm{~d} t}=-k^{2} T C_{k}+k q \frac{C_{1} C_{k-1}-C_{1}^{*} C_{k+1}}{2}+k(1-q) \frac{C_{2} C_{k-2}-C_{2}^{*} C_{k+2}}{2}$

We are interested in stability of a stationary solution, obtained according to (16), (17) or (18).

For the linear stability analysis, a small perturbation around stationary solution $\tilde{C}_{k}$ should be added, thus we set $C_{k}=\tilde{C}_{k}+c_{k}$ in equation (19), and in the first order in $c_{k}$ obtain 


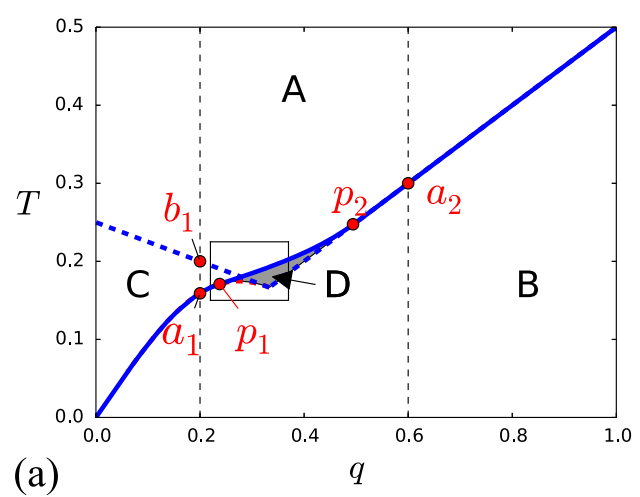

(a)

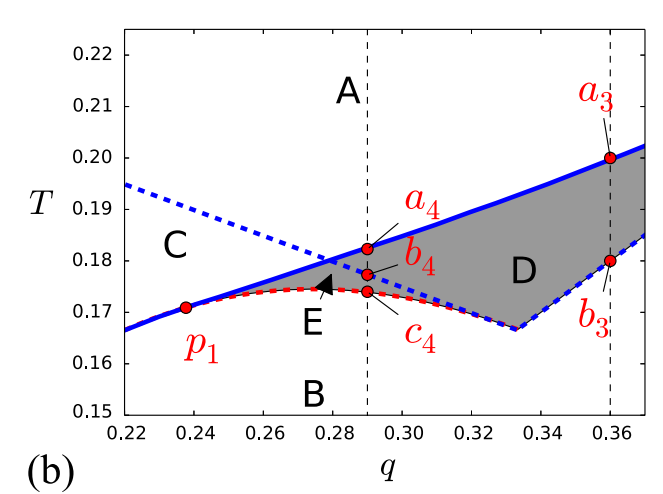

(b)

Figure 1. (a) Different regimes in the parameter plane $(q, T)$ are shown for $s=1$. Area A: asynchronous solution. Area B: coherent regime with $R_{1,2} \neq 0$. Area C: two-cluster coherent regime with only order parameter $R_{2} \neq 0, R_{1}=0$. Area $\mathrm{D}$ : region of bistability of incoherent and synchronous solutions. Area E: bistability of the twocluster state and a state with $R_{1,2} \neq 0$. Dashed blue lines are stability lines of the disordered state, obtained from (B11) and (B12). Between the points $p_{1}$ and $p_{2}$ the transition is hysteretic; dashed red line is the stability line of area C, obtained from (20), it coincides with the line where on the branch existing for small $T$ the first order parameter tends to zero. (b) Enlarged central region of panel (a). Vertical dashed lines are cuts of the diagram illustrated in figure 2 .

$$
\begin{aligned}
\frac{\mathrm{d} c_{k}}{\mathrm{~d} t}= & -k^{2} T c_{k}+\frac{k q}{2}\left(c_{1} \tilde{C}_{k-1}-c_{1}^{*} \tilde{C}_{k+1}+\tilde{C}_{1} c_{k-1}-\tilde{C}_{1}^{*} c_{k+1}\right) \\
& +\frac{k(1-q)}{2}\left(c_{2} \tilde{C}_{k-2}-c_{2}^{*} \tilde{C}_{k+2}+\tilde{C}_{2} c_{k-2}-\tilde{C}_{2}^{*} c_{k+2}\right) .
\end{aligned}
$$

Equation (20) is an infinite system, but because the amplitudes of modes with large $k$ tend to zero, it is possible to truncate it at some large $K$, and to write a finite system of equations (20), with $k$ varying from 1 to $K$, where $K$ is large enough. By finding a maximum eigenvalue of the corresponding matrix for the values of $q$ and $T$ of interest, it is possible to find stability properties of the solution $\tilde{C}_{k}$ and to build the boundary $q=q(T)$, where the solution $\tilde{C}_{k}$ changes its stability. This can be done both for general solutions (16), (17) and for the twocluster states (18).

We present the diagram of synchronous states in the parameter plane $(q, T)$, for the case of identical oscillators $s=1$ in figure 1. Here also the stability lines of the disordered state $\rho=(2 \pi)^{-1}$ are shown with dashed lines (see appendix B for details of calculation). Three major states can be observed: a disordered one, one with all non-zero order parameters, and a two-cluster one where all odd order parameters vanish. For small and large values of parameter $q$, i.e. where one of the coupling modes dominates (the first harmonics coupling for $q$ close to one or the second harmonics coupling for $q$ close to zero), the transitions are supercritical. We illustrate them in figure 2 (panels (a), (d)), showing dependencies of the order parameters on $T$. In the middle part of the phase diagram (between the points marked $p_{1}$, $p_{2}$ in figure 1), for $q$ close to 0.3 , the transitions are subcritical, so that a bistability occurs. These regimes are illustrated in figure 2 (panels (b), (c)). The transition from the disordered to the two-cluster state is always supercritical (see dashed red line in panels (c), (d) of figure 2). 

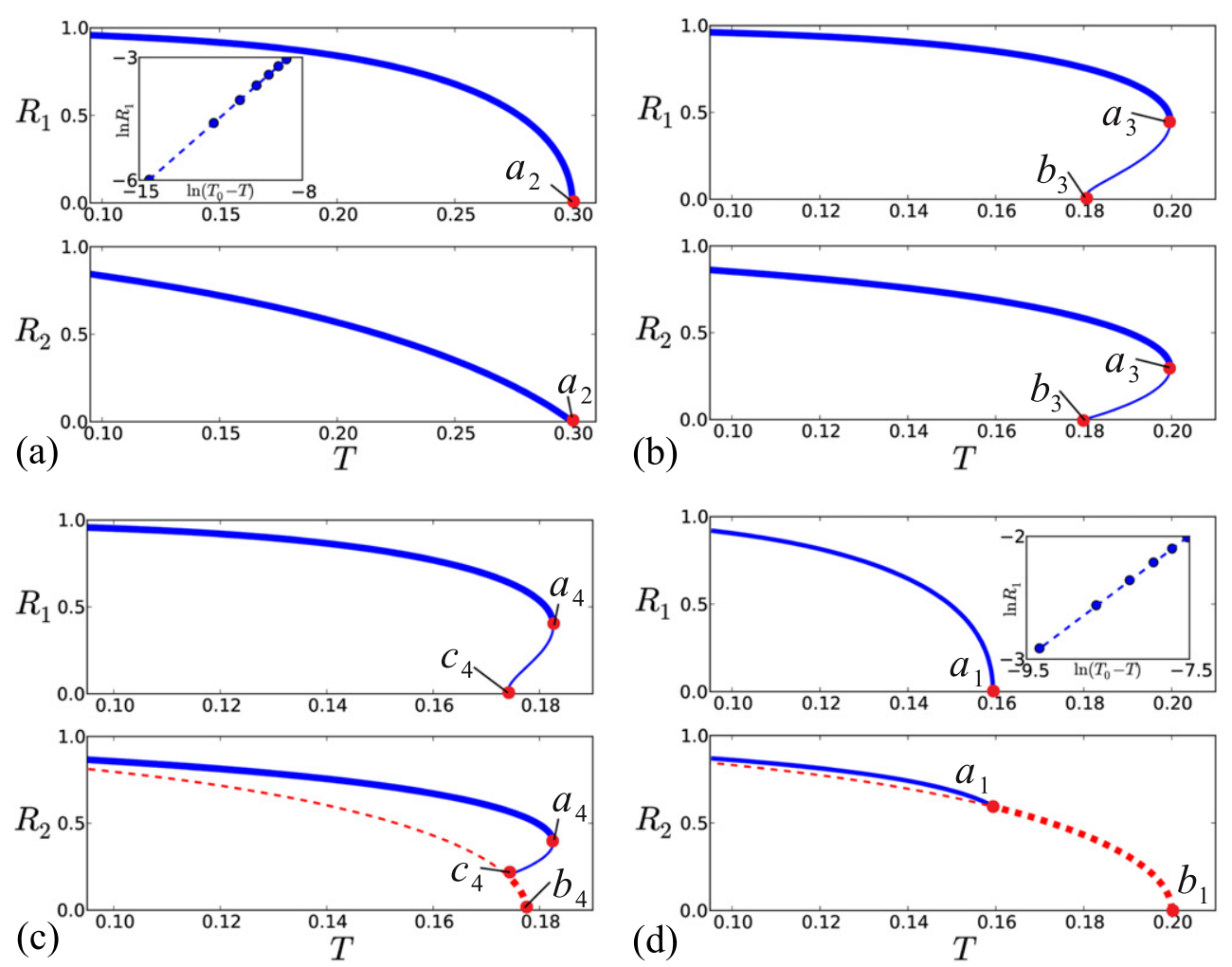

Figure 2. Dependencies of order parameters $R_{12}$ on the disorder parameter $T$, for $s=1$ and different values of $q$ : (a) $q=0.6$, (b) $q=0.36$, (c) $q=0.29$, (d) $q=0.2$. Solid blue line: branch of general solution $R_{1} \neq 0$; dashed red line: branch of the two-cluster state with $R_{1}=0, R_{2} \neq 0$. Thin curves depict unstable solutions, whereas the bold curves depict stable ones. Insets in the panels (a) and (d) present logarithmic plots indicating square-root dependencies of the order parameter $R_{1}$ on the criticality $T_{0}-T$ ( $T_{0}$ is a bifurcation point). Here markers denote solution of the self-consistent equations, the dashed line has a slope $1 / 2$.

\subsection{General phase diagram}

In this section we present the phase diagram for the unimodal symmetric distribution of natural frequencies (in calculations the Gaussian distribution $g(\omega)=(2 \pi)^{-1} \exp \left(-\omega^{2} / 2\right)$ was adopted). The lines of stability of the disordered state (bold dashed lines) are obtained according to stability analysis presented in appendix B, equations (B11), (B12). The phase diagrams on the plane of basic parameters $(q, T)$ for general values of $s$ are qualitatively the same as figure 1 . We show two cases $s=0.1$ (relatively weak noise, wide distribution of frequencies) and $s=0.5$ in figure 3 . All these diagrams are qualitatively similar, and we expect also that stability properties of different solutions are like in figure 2.

Remarkably, in the case of weak noise (figure 3(a)) the region of the first-order transition is significantly larger in parameter $q$, which governs the relation between two harmonics in the coupling function. On the contrary, for strong noise and narrow distribution of frequencies (figures 1,3(b)), first-order transitions are observed only in a small region of values of $q$, close to $q \approx 0.5$. 

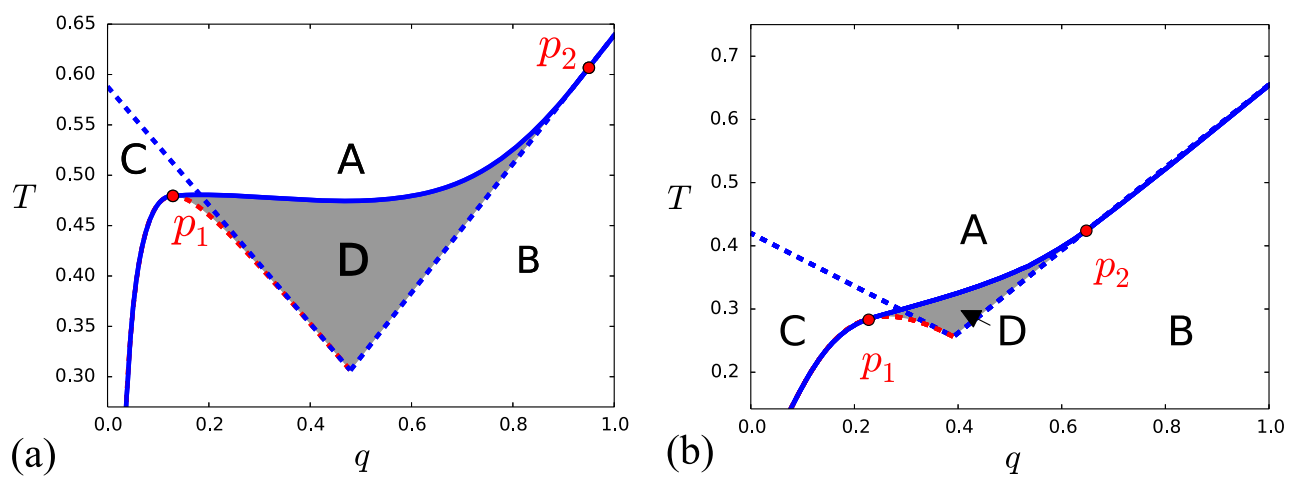

Figure 3. The same as figure 1, but for $s=0.1$ (a) and $s=0.5$ (b). Region $\mathrm{E}$ is not denoted because it is very tiny on these plots.
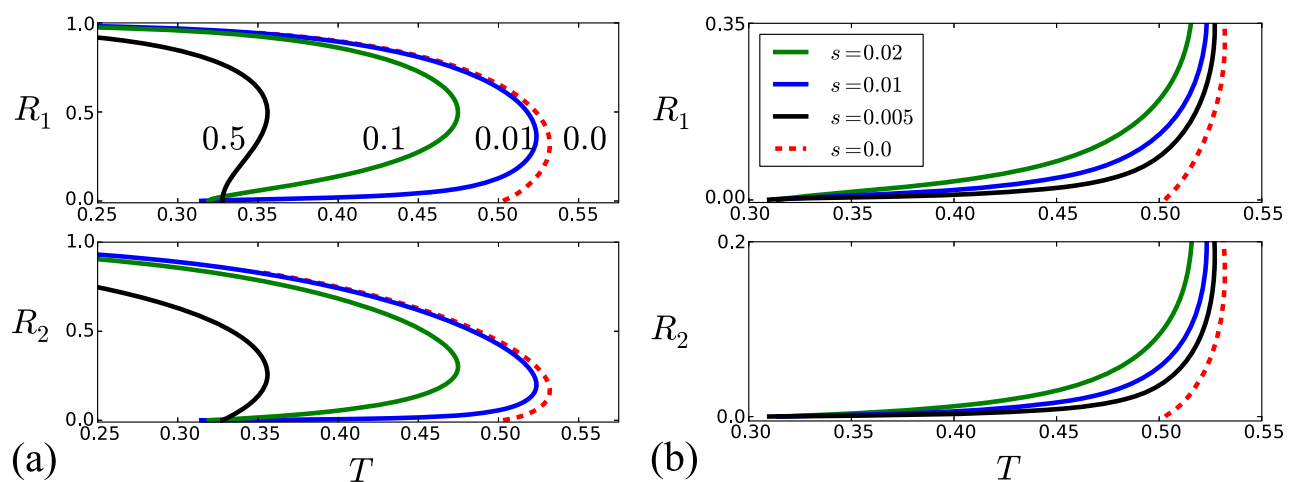

Figure 4. (a) The dependencies of order parameters $R_{1,2}$ on the overall disorder $T$ for $q=0.5$ and different values of $s$. The numbers on the upper panel depict values of $s$ : from the left curve to the right one $s$ changes from 0.5 to zero. The dashed curve corresponds to the special case of $s=0.0$. (b) The same as in (a) but now curves are plotted near the points of transition from incoherent to synchronous solutions $(R \rightarrow 0)$.

\section{Limiting case of small noise}

In this section we describe the limit of small noise $s \rightarrow 0$, which corresponds to the noise-free case of the bi-harmonic Kuramoto model studied in [7, 11, 12, 14, 16, 17]. This is the mostly challenging part, both numerically and theoretically. Numerically, there is a problem in finding a stationary probability density from (11), because this density becomes close to a delta-function, i.e. first a large number of Fourier modes is needed, and second the methods of solving an algebraic system for these modes converge very slowly. Theoretically, it is known that for a noise-free case, there is a multiplicity of solutions coexisting with a neutrally stable disordered state [14], this degeneracy is lifted by adding an arbitrary small noise. The reason for this singular limit is that the Fokker-Planck equation behaves singularly in the limit of small noise: while for any noise the stationary distribution is unique, in the noise-free case the order of equation is reduced and there are multiple solutions of the resulting Liouville equation. 
In order to shed light on the limit $s \rightarrow 0$, we fixed $q=0.5$, and calculated dependencies $R_{1,2}(T)$ for $s=0$ using the approach described in [7] for the noise-free case, and the approach above for different values of parameter $s$. The results presented in figure 4 show how the noise-free curve (dashed red curve in figure 4(a)) is approached as $s \rightarrow 0$ (solid curves in figure 4). Here a special discussion of how the noise-free solution has been obtained is needed. Indeed, the purely deterministic case $s=0$ is a singular limit of the Fokker-Planck equation, as in this limit the second derivative term vanishes. While the Fokker-Planck equation has a unique solution, there are multiple synchronous solutions which are characterized by different redistributions of locked oscillators between the stable branches in the noise-free case [7]. To select one of them that corresponds to the limit $s \rightarrow 0$, we use the following approach. Nontrivial multiple solutions of the noise-free problem occur if the effective potential for the motion of the phase has two minima on the interval $(0,2 \pi)$. For small noise, the probability density thus tends to a composition of two delta-functions at these minima. As for $\omega \neq 0$ (the case $\omega=0$ is less nontrivial as there the flux vanishes and the distribution density can be directly written as the exponent of the effective potential) the phase on average rotates, the amplitudes of these delta-functions are proportional to the lifetimes at the corresponding minima, or inversely proportional to the excitation rates for transitions from one minimum to another one. Thus, according to Kramers' formula for the excitation rates, one has to compare corresponding potential barriers, the steady state with the largest escape barrier will dominate in the limit of small noise. Thus, in the limit of small noise one gets rid of the multiplicity of states, by choosing 'the most stable' (in the sense of the largest excitation time) steady state. For the parameters of figure 4, this state is one which yields positive values of order parameter $R_{1}(\sigma=0$ in terms of the notations used in [7]); it has been used in calculations of the dashed red curve in figure 4(a).

One can see that there is a jump in the values of $T$ at which the curves touch the line $R_{1,2}=0$. For the case of noisy oscillators, these points are those where the disordered state loses its stability. For the noise-free case, it is another point, not related to a stability exchange. This jump is responsible for a formation of a 'boundary layer' (depicted in more details in figure 4(b)) where the noisy curves rapidly escape the vicinity of the curve $s=0$ as $R_{1,2}$ go to zero. It appears that the solution of the limiting case $s=0$ always differs significantly from the noisy solutions $s \neq 0$ at $R \rightarrow 0$, regardless of the noise strength represented by the parameter $s$.

In other words, the limit $s \rightarrow 0$ for the noisy case does not coincide with the noise-free solution $s=0$ near the bifurcation point $R=0$. This effect was also discussed in the papers $[7,14]$. In the limiting noise-free case $s=0$, the stationary solutions for distribution function $\rho(\varphi, \omega)$ are always singular: they contain a combination of delta-functions for any small $R_{1,2}$ (see [7] for detail). In contradistinction, the presence of the noise always regularizes solutions causing smooth and non-singular stationary distribution functions $\rho(\varphi, \omega)$. It is important to notice that the effective noise appears as a combination $s / R$ in the algebraic equations for stationary modes (14). Therefore, for any $s \neq 0$ we always have the limit of effectively 'large noise' at the bifurcation point $R \rightarrow 0$. The latter causes a qualitative difference near the transition point $R \rightarrow 0$ between noisy $(s \neq 0)$ and noise-free $(s=0)$ cases even for very small noise strength. With decrease of $s$, the 'boundary layer' shrinks (the smaller is parameter $s$, the smaller values $R$ we need for effective noise to be large) as one can see from figure 4 .

Remarkably, the incoherent state which is linearly stable in the thermodynamic limit can become metastable for finite-size ensembles in the region of bistability of asynchronous and synchronous solutions. As one can see from figures 4(a), (b), the lower branch of synchronous solutions (the unstable one) is relatively close to the incoherent state $R_{1,2}=0$ in the region $0.33 \lesssim T \lesssim 0.5$. Therefore, due to the random finite-size fluctuations, there is always a 


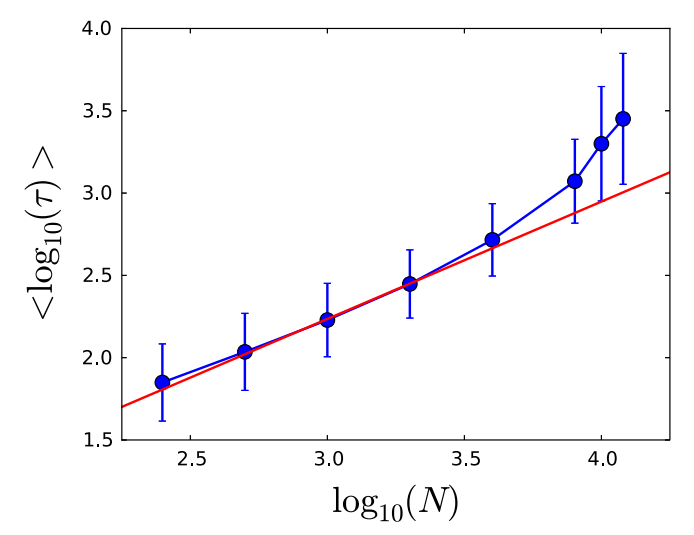

Figure 5. Statistics of the transition time from the incoherent state (linearly stable in thermodynamic limit) to synchronous mode for the finite size ensemble. Straight red line shows linear fit of the first five data points and has a slope $\sim 0.71$. Parameters: $q=0.5, T=0.47, s=0.01$.

probability for the system to escape the small basin of attraction of the disordered state, and to make a transition to the synchronous state which has a much larger basin. Figure 5 shows statistics of the lifetimes $\tau$ of the incoherent solution (linearly stable in thermodynamical limit) for relatively small noise strength $s=0.01$, and $T=0.47$. For each value of $N$ we performed many runs, at each of them the transition time $\tau$ was measured as the first time at which the order parameter $R_{1}$ exceeded value 0.5 (initially this parameter is close to zero). From these runs the mean value $\langle\log \tau\rangle$ and the standard deviation of $\log \tau$ have been calculated (so that the error bars in figure 5 are not due to insufficient averaging, but represent the variability of the times $\tau$ ).

The averaged time of transition increases as the number of oscillators in the system grows. Remarkably, one can see in figure 5(a) a crossover from a regime of a power-law dependence of the lifetime on the system size (for $N \lesssim 4000$ a relation $\tau \sim N^{0.71}$ holds) to a more rapid increase of the lifetime for $N \gtrsim 8000$. We have to compare this with the noise-free case, where according to [7] the lifetime scales as $\sim N^{0.72}$. One can see that for small system size, i.e. for large finite-size fluctuations, the noise-free situation is reproduced. We explain this as follows: small noise makes the asynchronous state only weakly stable, compared to neutral stability of the noise-free case, and for large finite-size fluctuations this weak stability is not seen. For smaller fluctuations (larger systems), the system starts to see the 'potential barrier' it needs to overcome to go into the stable state with a large order parameter. Now the lifetime is the activation time to escape from the stable state, which can be expected to follow an exponential Kramers' law for very small fluctuations (regime not accessible for moderate values of $N$ that we are able to study numerically). This explains the crossover observed in figure 5 .

\section{Conclusion}

The paper is devoted to the investigation of the Kuramoto model of globally coupled oscillators with bi-harmonic coupling function. In particular, here we concentrated on the effects caused by the action of the independent additive white noise forces on the oscillators. In the first part of the paper we have formulated the self-consistent theory that allows one to 
find stationary solutions for the system in the thermodynamic limit (when number of oscillators goes to infinity) in the presence of noise and an arbitrary distribution of frequencies. As a result of the developed theory, we calculated a general bifurcation diagram and described all possible stationary regimes of the model, for different values of noise strength, spread of frequencies distribution, and coupling constants.

In the noise-free case, as has been shown in [7, 14], there exists a multiplicity (multistability) of the coherent states due to the presence of the second harmonics in the coupling. In this work we have shown that the action of white noise withdraws the multiplicity, however the noise causes several additional complications to the bifurcation diagram.

First of all, due to the noise, the model contains a large area of parameters with so-called 'nematic phase', which represents a synchronous two-cluster state with zero first order parameter $\left(R_{1}=0, R_{2} \neq 0\right)$. Depending on the parameter values, the transitions to synchrony can be complicated, where possible scenarios include:

- a simple supercritical (second-order) transition to synchrony, where the first order parameter scales as a square root of supercriticality. This case is similar to the standard Kuramoto model with pure sinusoidal coupling and occurs when the second harmonics coupling is relatively small (figure 2(a));

- a subcritical (first-order) transition to synchrony, with a large area of bistability of coherent and asynchronous solutions. This type of transition becomes dominating as noise strength goes to zero and the second harmonics in the coupling function remains relatively strong (figure $2(\mathrm{~b})$ );

- first a two-cluster state appears via a supercritical (second-order) transition. This state is characterized by a zero first order parameter and a square-root scaling of the second order parameter. As disorder decreases, a general synchronous state appears via a next subcritical (first-order) transition. This happens in the case of relatively weak first harmonic in the coupling function (figure 2(c));

- first a two-cluster state appears via a supercritical (second-order) transition. As disorder decreases, a general synchronous state appears via a supercritical (second-order) transition, at which the amplitude of the first order parameter grows continuously. This happens in the case of very small first harmonics term in the coupling function (figure 2(d)).

We also report on the finite-size-induced transitions to synchrony for finite ensembles. As was mentioned before, in the thermodynamic limit the transition to synchrony can be hysteretic with a large area of bistability of synchronous and asynchronous solutions when the second harmonic is relatively large. In the latter case, noise causes a transition from incoherent state (linearly stable in the thermodynamic limit) to synchronous solution for finite-size ensembles.

Additionally, we would like to mention an analogy of our model of coupled oscillators with bi-harmonic coupling to an $X Y$-model of globally coupled spins with ferromagnetic and nematic coupling that is popular in statistical mechanics [18, 19]. In the latter context the desynchronized state is a disordered one, while one-cluster and two-cluster states are ferromagnetic and nematic ones. Two main differences are: (i) in the context of statistical mechanics, stability of solutions is established via minimization of the free energy in the canonical description or maximization of the entropy in the microcanonical one, while in the dynamical formulation above, stability properties are defined locally; (ii) diversity of oscillators' frequencies is a non-equilibrium feature not appearing in the equilibrium formulation of the $X Y$-model (see [20] for a comparison of the Kuramoto model with corresponding models from statistical physics). 
Finally, we would like to mention that in this paper we consider bi-harmonic coupling functions only. In general, a coupling function contains all harmonics, and one can ask whether addition of further harmonics changes the regimes described above. While such a study goes beyond the scope of this paper, we would like to mention that for narrow distributions of natural frequencies we expect a relatively small effect of higher harmonics. This expectation is based on the observation that for identical noisy oscillators the effect of the second harmonics term becomes significant for $q \approx 0.5$ only (see figure 1 ), where $1-q$ is the relative strength of the second harmonics term. Contrary to this, for a wide distribution of frequencies and relatively weak noise, the effect of the second harmonics is pronounced already for $q \approx 0.9$ (figure 3(a)). A detailed study of many-harmonics coupling will be presented elsewhere.

\section{Acknowledgments}

VV thanks the IRTG 1740/TRP 2011/50151-0, funded by the DFG/FAPESP. MK thanks Alexander von Humboldt foundation and the Russian Science Foundation (Project No. 14-1200811) for support. AP acknowledges the Galileo Galilei Institute for Theoretical Physics (Florence, Italy) for hospitality and the INFN for partial support during the completion of part of this work, and support by the grant (agreement 02..49.21.0003 of 27 August 2013 between the Russian Ministry of Education and Science and Lobachevsky State University of Nizhni Novgorod).

\section{Appendix A. General and symmetric solutions of the self-consistent equations}

We formulate here a more general problem, with coupling functions containing arbitrary phase shifts $\beta_{1}, \beta_{2}$ in coupling terms, compared to equation (6):

$$
\begin{aligned}
\frac{\mathrm{d} \varphi}{\mathrm{d} t}= & \omega(1-s) T+q R_{1} \sin \left(\Theta_{1}-\varphi-\beta_{1}\right)+(1-q) R_{2} \sin \left(\Theta_{2}-2 \varphi-\beta_{2}\right) \\
& +\sqrt{s T} \xi(t) .
\end{aligned}
$$

Equations for the order parameters are the same as equations (5):

$$
R_{m} \mathrm{e}^{\mathrm{i} \theta_{m}}=\left\langle\mathrm{e}^{\mathrm{i} m \varphi}\right\rangle=\iint g(\omega) \rho(\varphi, t \mid \omega) \mathrm{e}^{\mathrm{i} m \varphi} \mathrm{d} \varphi \mathrm{d} \omega .
$$

We look for solutions where the order parameters are stationary in the reference frame rotating with frequency $\Omega$ (yet unknown, to be defined in course of solution). Thus we introduce

$$
\Theta_{1}=\Omega t+\theta_{1}, \quad \Theta_{2}=2 \Omega t+\theta_{2}, \quad \varphi=\psi+\Theta_{1}-\beta_{1}
$$

to obtain

$$
\begin{aligned}
\frac{\mathrm{d} \psi}{\mathrm{d} t}= & \Omega+\omega(1-s) T+q R_{1} \sin (-\psi)+(1-q) R_{2} \sin \left(\theta_{2}-2 \theta_{1}-2 \psi-\beta_{2}+2 \beta_{1}\right) \\
& +\sqrt{s T} \xi(t),
\end{aligned}
$$


and

$$
\begin{aligned}
R_{1} \mathrm{e}^{\mathrm{i} \beta_{1}} & =\iint g(\omega) \rho(\varphi, t \mid \omega) \mathrm{e}^{\mathrm{i} \psi} \mathrm{d} \varphi \mathrm{d} \omega, \\
R_{2} \mathrm{e}^{\mathrm{i}\left(\theta_{2}-2 \theta_{1}+2 \beta_{1}\right)} & =\iint g(\omega) \rho(\varphi, t \mid \omega) \mathrm{e}^{\mathrm{i} 2 \psi} \mathrm{d} \varphi \mathrm{d} \omega .
\end{aligned}
$$

Let us denote $\delta=\theta_{2}-2 \theta_{1}+2 \beta_{1}-\beta_{2}$. Then we have a system

$\frac{\mathrm{d} \psi}{\mathrm{d} t}=\Omega+\omega(1-s) T+q R_{1} \sin (-\psi)+(1-q) R_{2} \sin (\delta-2 \psi)+\sqrt{s T} \xi(t)$,

and

$$
\begin{aligned}
R_{1} \mathrm{e}^{\mathrm{i} \beta_{1}} & =\iint g(\omega) \rho(\psi, t \mid \omega) \mathrm{e}^{\mathrm{i} \psi} \mathrm{d} \varphi \mathrm{d} \omega, \\
R_{2} \mathrm{e}^{\mathrm{i}\left(\delta+\beta_{2}\right)} & =\iint g(\omega) \rho(\psi, t \mid \omega) \mathrm{e}^{\mathrm{i} 2 \psi} \mathrm{d} \varphi \mathrm{d} \omega .
\end{aligned}
$$

The Fokker-Planck equation for $\psi$ reads

$\frac{\partial \rho}{\partial t}+\frac{\partial}{\partial \psi}\left[\left(\Omega+\omega(1-s) T+q R_{1} \sin (-\psi)+(1-q) R_{2} \sin (\delta-2 \psi)\right) \rho\right]=s T \frac{\partial^{2} \rho}{\partial \psi^{2}}$

and we are looking for stationary solutions (which are periodic with frequency $\Omega$ in the original reference frame). To find the solution of this self-consistent system explicitly, it is convenient to introduce two new auxiliary variables $R$ and $\alpha$ (together with definitions $u, v, x$, and $y$ ) according to

$$
\begin{aligned}
& R=\sqrt{\left(q R_{1} / T\right)^{2}+\left((1-q) R_{2} / T\right)^{2}}, \\
& u=\cos \alpha=\frac{q R_{1}}{T R}, \\
& v=\sin \alpha=\frac{(1-q) R_{2}}{T R}, \\
& x=\frac{\omega}{R}, \\
& y=\frac{\Omega}{T R} .
\end{aligned}
$$

Then the stationary Fokker-Planck equation (A4) for the stationary distribution density $\rho(\psi \mid x)$ (which depends on $R, \alpha, s, \delta, y$ as parameters) reads

$$
\frac{\partial}{\partial \psi}[R(y+x(1-s)-u \sin (\psi)-v \sin (2 \psi-\delta)) \rho]=s \frac{\partial^{2} \rho}{\partial \psi^{2}} .
$$

We are interested in the harmonics

$$
C_{n}(\alpha, R, s, x, \delta, y)=\int_{0}^{2 \pi} \rho \mathrm{e}^{-\mathrm{i} n \psi} \mathrm{d} \psi
$$


which allow to calculate the integrals in

$$
\begin{aligned}
& \iint g(\omega) \rho(\psi, t \mid \omega) \mathrm{e}^{\mathrm{i} \psi} \mathrm{d} \varphi \mathrm{d} \omega=R \int \mathrm{d} x g(R x) C_{1}^{*}(\alpha, R, s, x, \delta, y)=R F_{1}(\alpha, R, s, \delta, y), \\
& \iint g(\omega) \rho(\psi, t \mid \omega) \mathrm{e}^{\mathrm{i} 2 \psi} \mathrm{d} \varphi \mathrm{d} \omega=R \int \mathrm{d} x g(R x) C_{2}^{*}(\alpha, R, s, x, \delta, y)=R F_{2}(\alpha, R, s, \delta, y)
\end{aligned}
$$

and to find order parameters and parameters $\beta_{1,2}, T, q$ as functions of introduced quantities

$$
\begin{aligned}
R_{1} \mathrm{e}^{\mathrm{i} \beta_{1}} & =R F_{1}(\alpha, R, s, \delta, y), \\
R_{2} \mathrm{e}^{\mathrm{i}\left(\delta+\beta_{2}\right)} & =R F_{2}(\alpha, R, s, \delta, y), \\
T & =\frac{1}{\frac{R \cos \alpha}{R_{1}}+\frac{R \sin \alpha}{R_{2}}}, \\
q & =\frac{\frac{R \cos \alpha}{R_{1}}}{\frac{R \cos \alpha}{R_{1}}+\frac{R \sin \alpha}{R_{2}}}=\frac{1}{1+\frac{R_{1}}{R_{2}} \tan \alpha}, \\
\Omega & =y T R .
\end{aligned}
$$

We see that all the static parameters of the problem and the order parameters are explicit function of the four introduced auxiliary parameters $\alpha, R, \delta, y$. However, the corresponding analysis of the many-parameter space is rather tedious, therefore we restrict ourselves in the bulk of the paper to the simplest case where $\beta_{1}=\beta_{2}=0$ and symmetric distribution $g(\omega)$. One can see from the expressions above that such solutions can be obtained by setting $y=\delta=0$. Indeed, in this case $C_{1,2}(-x)=C_{1,2}^{*}(x)$ (see (14)) and quantities $F_{1,2}$ are real, so that indeed the conditions $\beta_{1}=\beta_{2}=0$ are satisfied and the frequency of the collective dynamics is the middle frequency of the distribution $\Omega=0$. We cannot exclude that there might be other asymmetric solutions with non-zero $y, \delta$, but we expect these to be nongeneric, as the conditions $\operatorname{Im}\left(F_{1}\right)=\operatorname{Im}\left(F_{2} \mathrm{e}^{-\mathrm{i} \delta}\right)=0$ can be fulfilled only occasionally.

\section{Appendix B. Stability analysis of the incoherent solution}

The detailed stability analysis of the incoherent solution of the system of phase equations with multi-harmonic coupling function has been performed in [21, 22]. Here we will present the analysis in the particular case of a bi-harmonic coupling function in the new parameter plane $(q, T, s)$.

Consider the following Fokker-Planck equation (7) for conditional probability density function $\rho(\varphi, t \mid \omega)$

$$
\frac{\partial \rho}{\partial t}+\frac{\partial}{\partial \varphi}\left[\left(\omega(1-s) T+q \operatorname{Im}\left(Z_{1} \mathrm{e}^{-\mathrm{i} \varphi}\right)+(1-q) \operatorname{Im}\left(Z_{2} \mathrm{e}^{-2 \mathrm{i} \varphi}\right)\right) \rho\right]=s T \frac{\partial^{2} \rho}{\partial \varphi^{2}}
$$

where

$$
Z_{m}(t)=\iint g(\omega) \rho(\varphi, t \mid \omega) \mathrm{e}^{\mathrm{i} m \varphi} \mathrm{d} \varphi \mathrm{d} \omega
$$


are the order parameters [15]. Then in the Fourier modes representation

$$
\rho(\varphi, t \mid \omega)=\frac{1}{2 \pi} \sum_{n} C_{n}(t, \omega) \mathrm{e}^{\mathrm{i} n \varphi}, \quad C_{n}(t, \omega)=\int_{0}^{2 \pi} \rho \mathrm{e}^{-\mathrm{i} n \varphi} \mathrm{d} \varphi, \quad C_{0}(t, \omega)=1
$$

we obtain

$$
\begin{aligned}
\frac{\mathrm{d} C_{k}}{\mathrm{~d} t}= & \int_{0}^{2 \pi}\left[-\frac{\partial}{\partial \varphi}\left[\left(\omega(1-s) T+q \operatorname{Im}\left(Z_{1} \mathrm{e}^{-\mathrm{i} \varphi}\right)+(1-q) \operatorname{Im}\left(Z_{2} \mathrm{e}^{-2 \mathrm{i} \varphi}\right)\right) \rho\right]\right. \\
& \left.+s T \frac{\partial^{2} \rho}{\partial \varphi^{2}}\right] \mathrm{e}^{-\mathrm{i} k \varphi} \mathrm{d} \rho=\left(-\mathrm{i} k \omega(1-s) T-k^{2} s T\right) C_{k} \\
& +\mathrm{i} k q \frac{Z_{1}^{*} C_{k-1}-Z_{1} C_{k+1}}{2 \mathrm{i}}+\mathrm{i} k(1-q)() \frac{Z_{2}^{*} C_{k-2}-Z_{2} C_{k+2}}{2 \mathrm{i}} .
\end{aligned}
$$

Thus we obtain the following system for $C_{k}$

$$
\begin{aligned}
\frac{\mathrm{d} C_{k}}{\mathrm{~d} t}= & k\left[-(\mathrm{i} \omega(1-\mathrm{s}) \mathrm{T}+\mathrm{ksT}) C_{k}+q \frac{Z_{1}^{*} C_{k-1}-Z_{1} C_{k+1}}{2}\right. \\
& \left.+(1-q) \frac{Z_{2}^{*} C_{k-2}-Z_{2} C_{k+2}}{2}\right]
\end{aligned}
$$

where

$$
Z_{1,2}(t)=\int g(\omega) C_{1,2}^{*}(t, \omega) \mathrm{d} \omega
$$

Small perturbation to incoherent solution $\rho(\varphi, t \mid \omega)=(2 \pi)^{-1}$ means that $C_{k} \ll 1$ for $k \neq 0$ and thus $Z_{m} \ll 1$, so in order to linearize the system (B5) we can neglect all the terms such as $C_{m} C_{n}$ and $Z_{m} C_{n}$ and higher. Then for $k>0$

$$
\begin{aligned}
\frac{\mathrm{d} C_{1}}{\mathrm{~d} t} & =-T(\mathrm{i} \omega(1-s)+s) C_{1}+q \frac{Z_{1}^{*}}{2} \\
\frac{\mathrm{d} C_{2}}{\mathrm{~d} t} & =-2 T(\mathrm{i} \omega(1-s)+2 s) C_{2}+(1-q) Z_{2}^{*}, \\
\frac{d C_{k}}{d t} & =-k T(\mathrm{i} \omega(1-s)+k s) C_{k}, \quad \text { for } k=3,4, \ldots
\end{aligned}
$$

and complex conjugate equations for $k<0$ because $C_{-k}=C_{k}^{*}$. Introduction to (B7) of the expressions (B6) for $Z_{1,2}$ gives integro-differential equations for $C_{1,2}$

$$
\begin{aligned}
& \frac{\mathrm{d} C_{1}}{\mathrm{~d} t}=-T(\mathrm{i} \omega(1-s)+s) C_{1}+q \frac{1}{2} \int g(\omega) C_{1} \mathrm{~d} \omega, \\
& \frac{\mathrm{d} C_{2}}{\mathrm{~d} t}=-2 T(\mathrm{i} \omega(1-s)+2 s) C_{2}+(1-q) \int g(\omega) C_{2} \mathrm{~d} \omega, \\
& \frac{\mathrm{d} C_{k}}{\mathrm{~d} t}=-k T(\mathrm{i} \omega(1-s)+k s) C_{k}, \quad \text { for } k=3,4, \ldots
\end{aligned}
$$

From (B8) it follows that the equations for the harmonics split and because $T \geqslant 0$ and $0 \leqslant s \leqslant 1$ all the high harmonics with $k \geqslant 3$ and their complex conjugates are stable, whereas instability appears in first and second harmonics independently, depending on $q, T$. 
Since the equations for modes (B8) are decoupled, in order to find boundary conditions when the first and second harmonics become unstable one should put in (B8) $\mathrm{d} C_{1} / \mathrm{d} t=0$ and $\mathrm{d} C_{2} / \mathrm{d} t=0$, and self-consistently obtain two conditions on the parameters $q, T$. Then, by using expression (B6) we obtain

$$
\begin{gathered}
T(\mathrm{i} \omega(1-s)+s) C_{1}=q \frac{1}{2} Z_{1}^{*}, \\
2 T(\mathrm{i} \omega(1-s)+2 s) C_{2}=(1-q) Z_{2}^{*} .
\end{gathered}
$$

Introducing (B9) to (B6)

$$
\begin{aligned}
& Z_{1}^{*}=\frac{q}{2 T} \int \frac{g(\omega) Z_{1}^{*}}{\mathrm{i} \omega(1-s)+s} \mathrm{~d} \omega, \\
& Z_{2}^{*}=\frac{1-q}{2 T} \int \frac{g(\omega) Z_{2}^{*}}{\mathrm{i} \omega(1-s)+2 s} \mathrm{~d} \omega,
\end{aligned}
$$

we obtain two lines on the $(q, T)$ parameter plane for any given $s$ :

$$
T=q \frac{1}{2} \int \frac{g(\omega) s}{\omega^{2}(1-s)^{2}+s^{2}} \mathrm{~d} \omega,
$$

and

$$
T=(1-q) \int \frac{g(\omega) s}{\omega^{2}(1-s)^{2}+4 s^{2}} \mathrm{~d} \omega,
$$

where the line (B11) on the $(q, T)$ plane corresponds to the linear stability boundary for $R_{1}=0$ and another line (B12) corresponds to the linear stability boundary for $R_{2}=0$.

\section{References}

[1] Kuramoto Y 1975 Int. Symp. on Mathematical Problems in Theoretical Physics (Lecture Notes in Physics vol 39) ed H Araki (New York: Springer) p 420

[2] Kuramoto Y 1984 Chemical Oscillations, Waves and Turbulence (Berlin: Springer)

[3] Acebròn J A, Bonilla L L, Vicente C J P, Ritort F and Spigler R 2005 Rev. Mod. Phys. 77137

[4] Ott E and Antonsen T M 2008 Chaos 18037113

[5] Ott E and Antonsen T M 2009 Chaos 19023117

[6] Czolczyński K, Perlikowski P, Stefański A and Kapitaniak T 2013 Commun. Nonlinear Sci. Numer. Simul. 18386

[7] Komarov M and Pikovsky A 2014 Physica D 28918

[8] Goldobin E, Koelle D, Kleiner R and Mints R G 2011 Phys. Rev. Lett. 107227001

[9] Kiss I Z, Zhai Y and Hudson J L 2005 Phys. Rev. Lett. 94248301

[10] Kiss I Z, Zhai Y and Hudson J L 2006 Prog. Theor. Phys. Suppl. 16199

[11] Daido H 1995 J. Phys. A: Math. Gen. 28 L151

[12] Daido H 1996 Phys. Rev. Lett. 771406

[13] Winfree A T 1980 The Geometry of Biological Time (Berlin: Springer)

[14] Komarov M and Pikovsky A 2013 Phys. Rev. Lett. 111204101

[15] Daido H 1996 Physica D 91 24-66

[16] Chiba H and Nishikawa I 2011 Chaos 21043103

[17] Li K, Ma S, Li H and Yang J 2014 Phys. Rev. E 89032917

[18] Teles T N, Benetti F P d C, Pakter R and Levin Y 2012 Phys. Rev. Lett. 109230601

[19] Pikovsky A, Gupta S, Teles T, Benetti F, Pakter R, Levin Y and Ruffo S 2014 Phys. Rev. E 90 062141

[20] Komarov M, Gupta S and Pikovsky A 2014 Europhys. Lett. 10640003

[21] Crawford J D 1995 Phys. Rev. Lett. 744341

[22] Crawford J D and Davies K T R 1999 Physica D 1251 